\title{
三元体系 $\mathrm{MgB}_{4} \mathrm{O}_{7}-\mathrm{MgSO}_{4}-\mathrm{H}_{2} \mathrm{O} 2^{\circ} \mathrm{C}$ 的研究 ${ }^{*}$
}

\author{
宋彭生 杜宪惠 孙 柏 \\ （中国科学院青海搵暗研究所，西宁）
}

作为盐湖卤水体系 $\mathrm{Li}^{+}, \mathrm{Mg}^{++} / \mathrm{Cl}^{-}, \mathrm{SO}_{4}^{--}, \mathrm{B}_{4} \mathrm{O}_{7}^{--}-\mathrm{H}_{2} \mathrm{O}$ 的次级体系，我们曾研究过 $\mathrm{Li}^{+}$I $\mathrm{B}_{4} \mathrm{O}_{7}^{--}, \mathrm{SO}_{4}^{--} \mathrm{H}_{2} \mathrm{O}, \mathrm{Li}^{+} / \mathrm{B}_{4} \mathrm{O}_{7}^{--}, \mathrm{SO}_{4}^{--}, \mathrm{Cl}^{-}-\mathrm{H}_{2} \mathrm{O} 25^{\circ} \mathrm{C}$ 时的相关系与溶液的物化性质 ${ }^{[1,2]}$. 本 文报道对含鋘的三元体系 $\mathrm{MgB}_{4} \mathrm{O}_{7}-\mathrm{MgSO}_{4}-\mathrm{H}_{2} \mathrm{O} 25^{\circ} \mathrm{C}$ 的研究结果。

\section{一、实 检 部 分}

1. 原科、实验和分析方法实验中所用的硫酸镁为 A.R. 级试剂 $\mathrm{MgSO}_{4} \cdot 7 \mathrm{H}_{2} \mathrm{O}$ 经一次 国结晶, 空气中风干后使用. 四砽酸镁 $\left(\mathrm{MgB}_{4} \mathrm{O}_{7}: 9 \mathrm{H}_{2} \mathrm{O}\right)$ 系实验室合成产物, 化学成分为 (直量\%): $\mathrm{MgO} 11.83, \mathrm{~B}_{2} \mathrm{O}_{3} 40.76, \mathrm{H}_{2} \mathrm{O} 47.43, \mathrm{~B}_{2} \mathrm{O}_{3} / \mathrm{MgO}$ (摩尔比) 1.995 (理论值分别 为 $11.80,40.75,47.45,2.000)$. 经 X-射线粉晶衍射、热分析和红外吸收光谱鉴定与章氏研 镜石 (Hungtsa oite, $\mathrm{MgB}_{4} \mathrm{O}_{7} \cdot 9 \mathrm{H}_{2} \mathrm{O}$ ) 一致.

体系的研究是采用等温溶解法进行的. $2^{\circ} \mathrm{C} \mathrm{MgB} 4 \mathrm{O}_{7} \cdot 9 \mathrm{H}_{2} \mathrm{O}$ 在水中溶解时, 经一周连续 强烈摚拌后, 会向 $\mathrm{Mg}_{2} \mathrm{~B}_{6} \mathrm{O}_{\mathfrak{u}} \cdot 15 \mathrm{H}_{2} \mathrm{O}$ (多水硼镁石, Inderite) 转化. 因为在 $25^{\circ} \mathrm{CMgO}-\mathrm{B}_{2} \mathrm{O}_{3}$ -

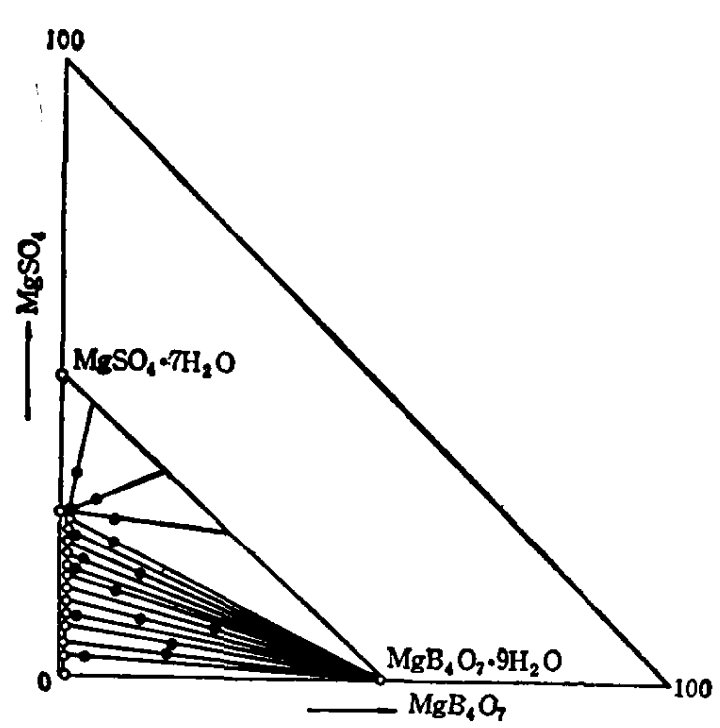

图 1 三元体系 $\mathrm{MgB}_{4} \mathrm{O}_{7}-\mathrm{MgSO}_{4}-\mathrm{H}_{2} \mathrm{O}_{25} 5^{\circ}$ 溶解 度等温图

$\mathrm{H}_{2} \mathrm{O}$ 体系中, $\mathrm{MgB}_{4} \mathrm{O}_{7} \cdot 9 \mathrm{H}_{2} \mathrm{O}$ 是一种不相称溶 解的固相。但当溶液中有 $\mathrm{MgSO}_{4}$ 存在且达到 一定浓度时, 可以阻滞这种转化. 这才使得本 三元体系的等温研究成为可能. 本文中三元系 所有的液相组成都是在约半月之内经 $2 \sim 3$ 次 取样确认组成不变时的浓度值. $\mathrm{MgB}_{4} \mathrm{O}_{7} \cdot 9 \mathrm{H}_{2} \mathrm{O}$ 在纯水中的溶解度是于一周内经数次取样测得 的未发生转化时的介稳平衡浓度值.

液固相样品中的硼是在甘露醇存在下以碱 量法测定, 以 $\mathrm{B}_{2} \mathrm{O}_{3}$ 表示的. $\mathrm{MgO}$ 以甲基红为 指示剂酸量法测定. 总是同时分别测定液固相 样品中的 $\mathrm{B}_{2} \mathrm{O}_{3}$ 和 $\mathrm{MgO}$ 浓度, 计算 $\mathrm{B}_{2} \mathrm{O}_{3} / \mathrm{MgO}$ 摩尔比, 以考查溶解过程中 $\mathrm{MgB}_{4} \mathrm{O}_{7} \cdot 9 \mathrm{H}_{2} \mathrm{O}$ 是 否发生转化。

液相性质的测定方法与前文相同 ${ }^{[1]}$ 。溶解度及液相性质测定是在 $25 \pm 0.03^{\circ} \mathrm{C}$ 恒温下进引。

2. 实验结果 三元系 $\mathrm{MgB}_{4} \mathrm{O}_{7}-\mathrm{MgSO}_{4}-\mathrm{H}_{2} \mathrm{O} 25^{\circ} \mathrm{C}$ 溶解度和液相性质的测定结果列在 表 1 中. 图 1 为体系的 $25^{\circ} \mathrm{C}$ 溶解度等温图. $25^{\circ} \mathrm{C}$ 溶解度等温线由两条溶解度分支组成, 分别 对应于 $\mathrm{MgB}_{4} \mathrm{O}_{7} \cdot 9 \mathrm{H}_{2} \mathrm{O}$ 和 $\mathrm{MgSO}_{4} \cdot 7 \mathrm{H}_{2} \mathrm{O}$ 的结晶区. 共饱点组成为: $\mathrm{MgB}_{4} \mathrm{O}_{7} 1.02 \%, \mathrm{MgSO}_{4}$ $26.54 \%$. 在三元系中，没有发生原始组份的脱水作用，也没有形成复盐或固溶体.

本文 1986 年10月23日收到。

*国家自然科学荎金资助项目. 


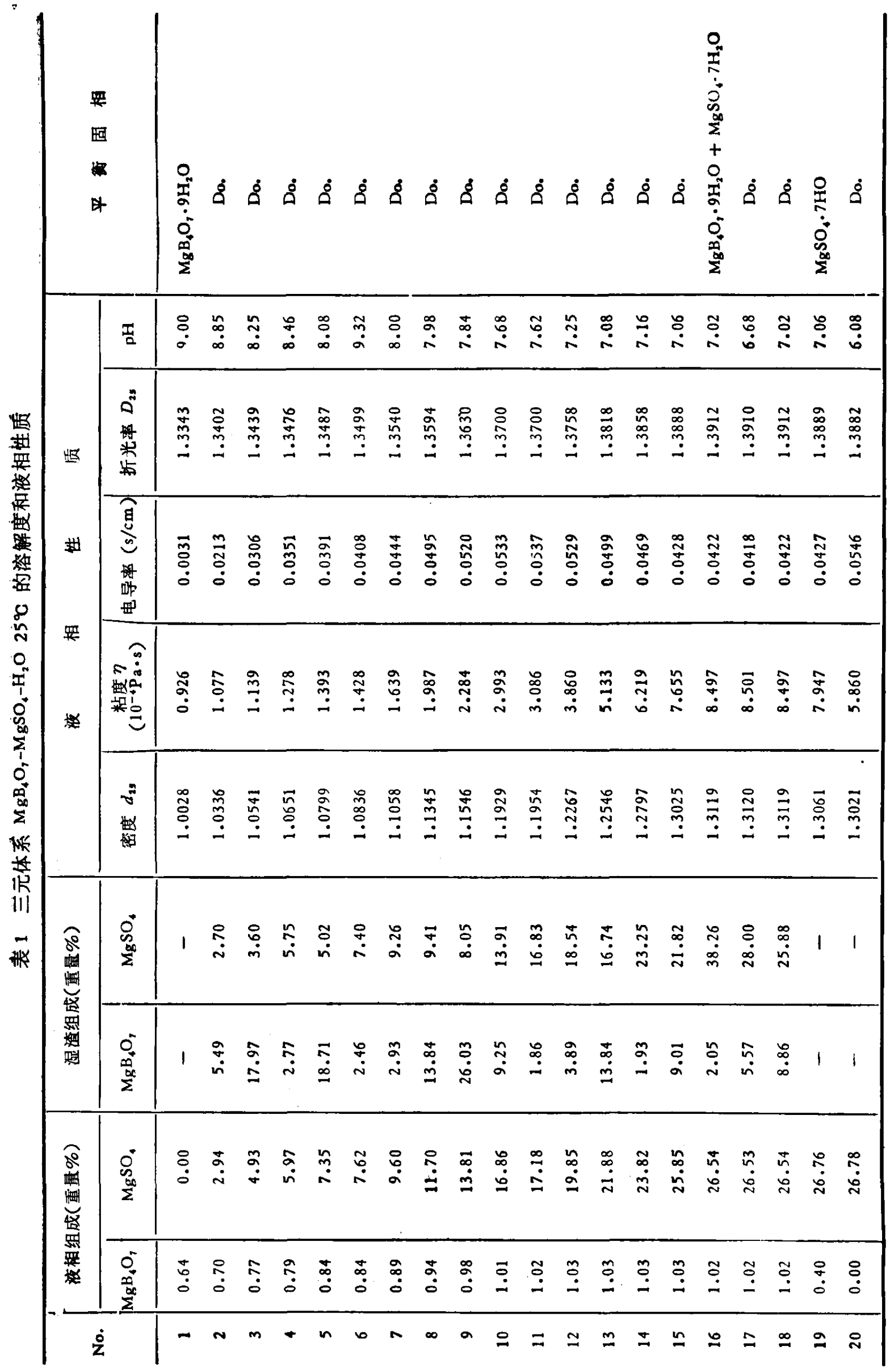




\section{二、讨 论}

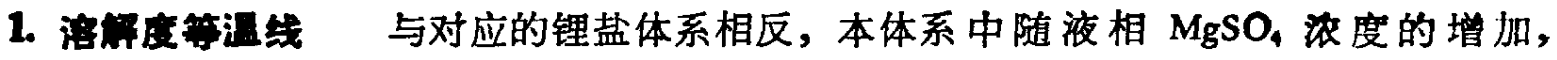
$\mathrm{MgB}_{4} \mathrm{O}_{7}$ 的浓度变大,即 $\mathrm{MgSO}_{4}$ 对后者有盐溶作用. 对这种相互作用关系,目前尚难以用机 理模型进行研究。

以重摩浓度 $m$ 表示的体系中 $\mathrm{MgB}_{4} \mathrm{O}$ ，的溶解度线，可以拟合成下面的二次方程:

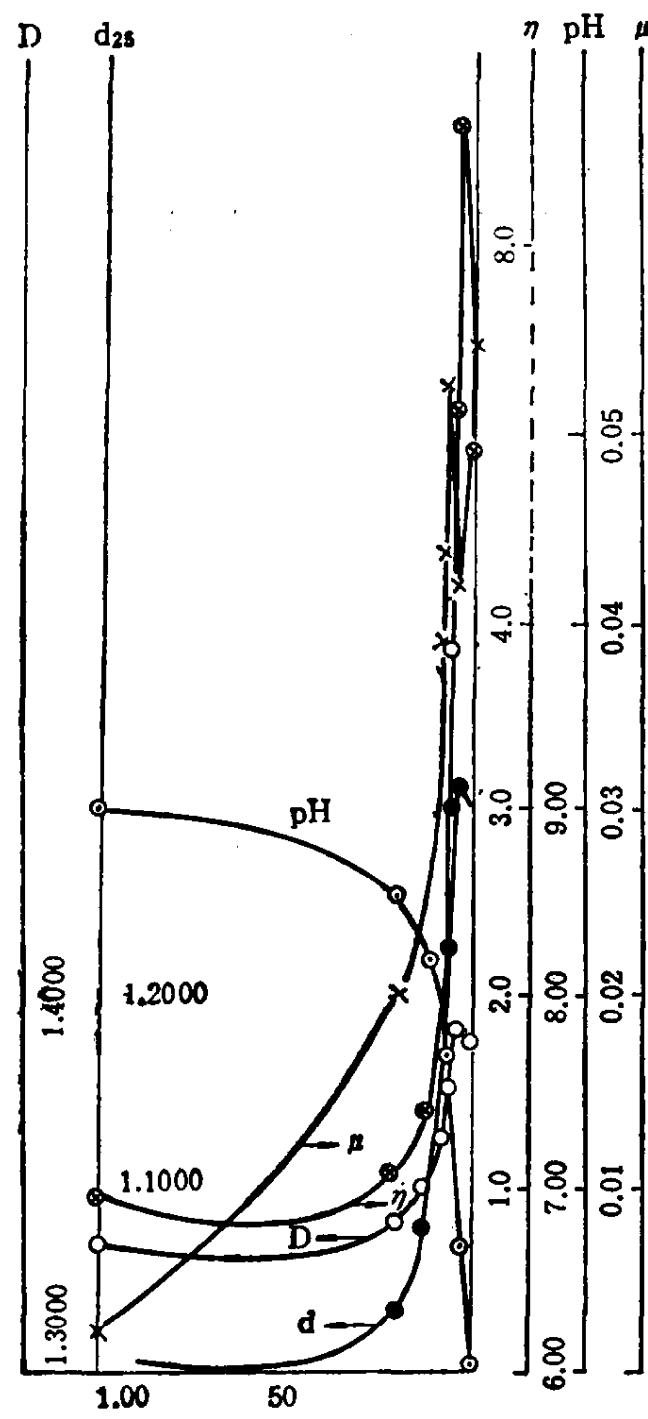

图 2 三元体系 $\mathrm{MgB}_{4} \mathrm{O}_{4}-\mathrm{MgSO}_{4}-\mathrm{H}_{2} \mathrm{O} 25^{\circ} \mathrm{C}$ 组成生质图

$$
\begin{aligned}
m_{\mathrm{MgB}_{4} \mathrm{O}_{7}}=a_{0} & +a_{1} \times m_{\mathrm{MgSO}_{4}}+a_{2} \\
& \times\left(m_{\mathrm{MgSO}_{4}}\right)^{2},
\end{aligned}
$$

式中 $a_{0}=0.03539, a_{1}=0.02621$,

$$
a_{2}=-0.00399 \text {. }
$$

计算值与测定值的对比列在表 2 中, 偏差的绝对 值皆不超过 $0.001 \mathrm{~m}$.

2. 洝波的性质图 2 为三组份体系 $\mathrm{MgB}_{4} \mathrm{O}_{7}-\mathrm{MgSO}_{4}-\mathrm{H}_{2} \mathrm{O} 25^{\circ} \mathrm{C}$ 时的组成-性质图. 随 液相组成的变化, 液相的性质也平滑地改变. 图 上的奇异点对应于体系的共饱点.

我们仍可采用与锂盐体系类似的公式计算体 系中饱和溶液的密度:

$$
\begin{gathered}
\ln \frac{d_{25}}{d_{25}^{\omega}}=a \times \mathrm{MgB}_{4} \mathrm{O}_{7} \% \\
+b \times \mathrm{MgSO}_{4} \%,
\end{gathered}
$$

系数 $a=8.77 \times 10^{-3}, b=9.97 \times 10^{-3}, d_{25}^{20}$ 为 纯水在 $25^{\circ} \mathrm{C}$ 时的密度, 其值为 0.99707 . 计算结 果列在表 2 中,最大偏差 $0.3 \%$, 同样, 本文仍使用 我们以前提出的公式计算溶液的折光率 $D_{25}$ :

$$
\begin{aligned}
\ln \frac{D_{25}}{D}= & a_{1} \times \mathrm{MgB}_{4} \mathrm{O}_{7} \%+b_{1} \\
& \times \mathrm{MgSO}_{4} \%,
\end{aligned}
$$

式中系数 $a_{1}=4.73 \times 10^{-4}, b_{1}=1.49 \times 10^{-3}$, 常数 $D-1.33389$. 计算值的最大偏差为 $0.2 \%$, 结果也列在表 2 中.

在 $\mathrm{MgSO}_{4}$ 存在下 $\mathrm{MgB}_{4} \mathrm{O}_{7} \cdot 9 \mathrm{H}_{2} \mathrm{O}$ 溶解达到饱 和时, 其活度积应为常数, 即

$$
K=\left(m_{\mathrm{Mg}}++\times m_{\mathrm{B}_{4} \mathrm{O}_{7}^{-}}^{-}\right) \times r_{ \pm}^{2} \times a_{w}^{9},
$$

或以对数表示则有

$$
\ln K=\ln \left(m_{\mathrm{Mg}^{++}} \times m_{\mathrm{B}_{4} \mathrm{O}_{7}^{--}}\right)+2 \ln \left(r_{ \pm} \cdot a_{\omega}^{9 / 2}\right),
$$

式中 $m_{\mathrm{Mg}}^{++}, m_{\mathrm{B}_{4} \mathrm{O}_{7}^{--}}$一溶液中 $\mathrm{Mg}^{++}$和 $\mathrm{B}_{4} \mathrm{O}_{7}^{--}$离子的化学计量重摩浓度, $r_{ \pm}$一溶液中 $\mathrm{MgB}_{4} \mathrm{O}_{7}$ 的平均活度系数, $a_{N}$ 一溶液中水的活度. 考虑到通常的图解外推至 $\sqrt{I}=0$ 的 方法误差较大，我们改用下法处理: 选取 $\mathrm{MgSO}_{4}$ 浓度为 0 和另一很小浓度 (例如 $0.0005 \mathrm{~m}$ ) 
表 2 计算的溶解度及溶液密度折光率与实测值的对比

\begin{tabular}{|c|c|c|c|c|c|c|c|c|c|c|}
\hline No. & $\underset{m}{\mathrm{MgSO}_{4}}$ & $\begin{array}{c}\text { 实测的 } \\
\mathrm{MgB}_{4} \mathrm{O}_{7} m\end{array}$ & $\begin{array}{c}\text { 计算的 } \\
\mathrm{MgB}_{4} \mathrm{O}_{7} m\end{array}$ & 差 & $\begin{array}{c}\text { 实测的 } \\
d_{23}\end{array}$ & 计算的 & 差 & $\mid \begin{array}{c}\text { 实测的 } \\
D_{29}\end{array}$ & $\left|\begin{array}{c}\mathrm{T}_{13} \\
D_{25}\end{array}\right|$ & 差 \\
\hline 1 & 0.000 & 0.036 & 0.035 & -0.001 & 1.0028 & 1.0028 & 0.0000 & 1.3343 & 1.3343 & 0.0000 \\
\hline 2 & 0.025 & 0.041 & 0.042 & +0.001 & 1.0336 & 1.0331 & -0.0005 & 1.3402 & 1.3402 & 0.0000 \\
\hline 3 & 0.434 & 0.046 & 0.046 & 0.000 & 1.0541 & 1.0544 & +0.0003 & 1.3439 & 1.3439 & 0.0000 \\
\hline 4 & 0.532 & 0.047 & 0.048 & +0.001 & 1.0651 & 1.0656 & +0.0005 & 1.3476 & 1.3463 & -0.0013 \\
\hline 5 & 0.665 & 0.051 & 0.051 & 0.000 & 1.0799 & 1.0808 & +0.0009 & 1.3487 & 1.3491 & +0.0004 \\
\hline 6 & 0.692 & 0.051 & 0.052 & +0.001 & 1.0836 & 1.0837 & +0.0001 & 1.3499 & 1.3497 & -0.0002 \\
\hline 7 & 0.891 & 0.055 & 0.056 & +0.001 & 1.1058 & 1.1058 & 0.0000 & 1.3540 & 1.3537 & -0.0003 \\
\hline 8 & 1.113 & 0.060 & 0.060 & 0.000 & 1.1345 & 1.1297 & -0.0048 & 1.3594 & 1.3580 & -0.0014 \\
\hline 9 & 1.346 & 0.064 & 0.063 & -0.001 & 1.1546 & 1.1541 & -0.0005 & 1.3630 & 1.3623 & -0.0007 \\
\hline 10 & 1.705 & 0.069 & 0.068 & -0.001 & 1.1929 & 1.1901 & -0.0028 & 1.3700 & 1.3685 & -0.0015 \\
\hline 11 & 1.745 & 0.069 & 0.069 & 0.000 & 1.1954 & 1.1940 & -0.0014 & 1.3700 & 1.3691 & -0.0009 \\
\hline 12 & 2.084 & 0.073 & 0.073 & 0.000 & 1.2267 & 1.2263 & -0.0004 & 1.3758 & 1.3746 & -0.0012 \\
\hline 13 & 2.358 & 0.074 & 0.075 & +0.001 & 1.2546 & 1.2514 & -0.0032 & 1.3818 & $\mid 1.3788$ & -0.0030 \\
\hline 14 & 2.633 & 0.076 & 0.077 & +0.001 & 1.2797 & 1.2759 & -0.0038 & 1.3858 & 1.3828 & -0.0030 \\
\hline 15 & 2.937 & 0.079 & 0.078 & -0.001 & 1.3025 & 1.3019 & -0.0006 & 1.3888 & 1.3869 & -0.0019 \\
\hline 16 & 3.044 & 0.078 & 0.078 & 0.000 & 1.3119 & 1.3108 & -0.0011 & 1.3912 & 1.3884 & -0.0028 \\
\hline 17 & 3.042 & 0.078 & 0.078 & 0.000 & 1.3120 & 1.3107 & -0.0013 & 1.3910 & 1.3883 & -0.0027 \\
\hline 18 & 3.044 & 0.078 & 0.078 & 0.000 & 1.3119 & 1.3108 & -0.0011 & 1.3912 & 1.3884 & -0.0028 \\
\hline 19 & 3.052 & 0.031 & - & - & 1.3061 & 1.3065 & +0.0004 & 1.3889 & 1.3884 & -0.0005 \\
\hline 20 & 3.0386 & - & - & - & 1.3021 & 1.3022 & +0.0001 & 1.3882 & 1.3882 & 0.0000 \\
\hline
\end{tabular}

两个点,按式(1)分别算出 $\mathrm{MgB}_{4} \mathrm{O}_{7}$ 的对应浓度及 $\ln \left(m_{\mathrm{Mg}^{+}}+\times m_{\mathrm{B}_{4} \mathrm{O}_{7}^{--}}\right)$和 $2 \sqrt{I}$, 然后求通过 此两点的直线方程, 及与 $\mathrm{y}$ 轴的截距, 即 $\ln K$. 所得数值为 $\ln K--8.73$. 我们又采用另一 种方法,接照 $D_{\text {avies }}$ 公式 ${ }^{(4)}$ 计算 $\mathrm{MgB}_{4} \mathrm{O}_{7}$ 的饱和水溶液 $0.0358 \mathrm{~m}$ )中的活度系数和水活度,计 算结果为 $r_{ \pm}=0.344, a_{n}-0.9995$, 故 $K=(0.0358 \times 0.344)^{2} \times 0.9995^{9}, \ln K=-8.80$. 此值与第一种方法所得结果很一致. 取二者的算术平均值 $-8.765 \pm 0.035$ 作为 $\ln K$ 值.

$\mathrm{MgB}_{4} \mathrm{O}_{7} \cdot 9 \mathrm{H}_{2} \mathrm{O}$ 在水中溶解过程:

$$
\mathrm{MgB}_{4} \mathrm{O}_{7} \cdot 9 \mathrm{H}_{2} \mathrm{O}=\mathrm{Mg}^{++}+\mathrm{B}_{4} \mathrm{O}_{7}^{--}+9 \mathrm{H}_{2} \mathrm{O}
$$

的自由能变化为

$$
\Delta G^{\circ}--R T \ln K .
$$

根据文献 [5], 式(6)右方三者的标准生成自由能分别为 $-455.10,-2606.70,-637.30 \mathrm{KJ} /$ $\mathrm{mol}$. 如以 $G_{\mathrm{Hung}}^{\circ}$ 代表章氏硼镁石的标准生成自由能,于是

$$
\begin{gathered}
\Delta G^{\circ}--108.70-622.60-56.679 \times 9-G_{\text {Hung }}^{\circ}=-1241.411-G_{\text {Hung }}^{\circ}, \\
G_{\text {Hung }}^{\circ}=-1241.411+R T \ln K=-1246.60 \pm 0.02 \mathrm{kcal} / \mathrm{mol}=-5215.77 \pm 0.08 \mathrm{~kJ} / \mathrm{mol} .
\end{gathered}
$$

\section{$\Rightarrow$ 文 触}

[1] 宋彭生、杜宪惠、许佰存,科学通报,28(1983),2: 106-110.

[2] 宋彭生、杜宪惠,科学通报, 31(1986), 3: 209-213.

[3] Валяшко М.Г. итд., Ж. Неоре. Х., 5(1960), 1316-1328.

[4] 黄子卿,电解质溶液理论导论(修订本), 科学出版社, 1983 .

[5] Wagman, D. D. et al., Selected values of chemicul thermodynamic properties, NBS Technical Note 1968, $270-273$. 